wahrscheinlich mehr Realitätssinn als diejenigen, die den Gefühlen des Zeitgeistes nachhängen. Er weiß wohl, daß mit jeder Anerkennung hohe Souveränitätskosten entstehen, wahrliche »faux frais « (Marx), auf deren globale Übernahme als Recht auf Entwicklung gepocht wird. Wenn er die Werte des Sozialismus anmahnt (womit ja wahrscheinlich nicht der Leninismus gemeint ist), dann -- so nehme ich an geschieht das in der Erfahrung, daß die aktuellen a-nationalen Politik-Standards, wie sie von IWF und Weltbank gesetzt werden, in ihrer ideologischen Fixierung auf die $>$ Wahrheit des Marktes< weit hinter den sozialen und politischen Errungenschaften herhinken, für die Gewerkschaften und sozialistische Parteien gekämpft haben, und die doch auch zu den allgemeinen Produktionsbedingungen der bürgerlichen Gesellschaft gehören. Dies läßt sich in neuen oder alten Staaten nicht (mehr) durchsetzen, sondern nur in der a-nationalen Politik einer neuen Weltordnung.

\title{
Michael Daxner Die Überführung der Wissenschaft in den Westen
}

\begin{abstract}
》Ich schweige zu diesen Unıversitätsabwicklungen. Mir sind sie als Verwaltungsakt zuwider, und ich sehe da Ritterheere mit Rüstung, Stıefeln und Sporen einretten und die Lehen neu verteilen. Ostgotischer Patriotismus erfüllt mıch mit Empörung, und ich habe den Impuls, zum Schwert zu greifen und unser geistiges Eigentum vor dem Eindringen zu schützen. Andererseits erinnere ich mich an die Herrscha.ftsarroganz, die früher von den gesellschaftswissenschaftlichen Fakultäten ausging. Ich will Namen und Orte hier nicht nennen, habe aber mehrere Fälle im Gedächtnıs, wo die "prinzıpıenfesten" Kollegen einen der Ihren gnadenlos zerfetzten und ausstießen, der argenwie mit der Linie in Konflikt gekommen war. Und jetzt nennen sıe sich nıcht mehr Marxismus-Leninısmus, sondern Friedens- oder Zivilisationsforschung oder irgendwe ähnlich harmlos; aber auf der Bühne sind bis auf eın paar geopferte Vorruheständler die alten Haudegen, und in demokratischer Tugend bestätıgen sıe einem der Ihren zu mehr als Zweidrıtteln das politische Vertrauen: Universı̈ätsautonomıe. " ${ }^{\mathrm{t}}$
\end{abstract}

\section{Die Anergnung des Fremden}

Darin ist schon vieles vorweggenommen. Die Überführung des Wissenschaftssystems der Deutschen Demokratischen Republik in das der Bundesrepublik Deutschland ist kein grundsätzlich rechtliches Problem. Die rechtsstaatliche Legitzmatıon dieser wissenschaftlich, sozial, kulturpolitısch und rechtlich gleichermaßen zweifelhaften Prozedur legt allerdings exemplarisch offen, welche Folgen die Einverleibung eines Staates durch einen anderen hat. Die Bundesrepublik hat sich einen anderen Staat angeeignet, inkorporiert. Ulrich K. Preuß' Definition trifft den Sachverhalt besser als »Anschluß«, »Kolonisierung« oder gar »Wiedervereinigung«: "Der Vorgang, mit dem wir es bei der Vereinigung der beiden deutschen Staaten zu tun haben, ist nicht der einer durch Vertrag begründeten Staatenverbindung in der

I Jens Reich, Buridans Esel, in: Constructiv 2/1991, S. [4-15. 
Form einer Staatsgründung, ein politischer Einigungsvertrag mithin, sondern eine technisch ziemlich perfekt organisierte Staatennachfolge: die Bundesrepublik tritt die Nachfolge der Gebietsherrschaft der DDR an. Sie ist, wie man sagt, der ,Erwerberstaat .... $\ll^{2}$

Wissenschaftlich ist die Frage zu entscheiden, ob zwei Systeme, die in der Struktur inkompatibel sind, aber innerwissenschaftlich bloß Differenzen bilden, durch Fusion unter dem Diktat des einen Systems den nationalen Interessen an Wissenschaft genügen. Trifft die Präambel von Artikel 38 Einıgungsvertrag zu, "Wissenschaft und Forschung bilden auch im vereinten Deutschland wichtige Grundlagen für Staat und Gesellschaft. Der notwendigen Erneuerung von Wissenschaft und Forschung unter Erhaltung leistungsfähiger Einrichtungen in dem in Artikel 3 genannten Gebiet dient eine Begutachtung von öffentlich getragenen Einrichtungen durch den Wissenschaftsrat, die bis zum 31. Dezember 1991 abgeschlossen sein wird, wobei einzelne Ergebnisse schon vorher schrittweise umgesetzt werden sollen, « ${ }^{3}$ dann ist die semantische Differenz zur programmatischen Aussage des Wissenschaftsrates zu klären: "Wissenschaftliche Forschung, d.h. methodisch geleiteter Erkenntnisgewinn, ist zu einem konstitutiven Bestandteil der Kultur der heutigen Gesellschaft geworden. Das gilt sowohl für die Natur- und Ingenieurwissenschaften als auch für die Geistes- und Sozialwissenschaften. Die wissenschaftliche Forschung bildet eine wesentliche Voraussetzung für die gesellschaftliche, wirtschaftliche und kulturelle Dynamik.

Für eine hochentwickelte Industriegesellschaft ohne nennenswerte natürliche Rohstoffe hat eine leistungsfähige und im internationalen Wettbewerb konkurrenzfähige Forschung eine besondere Bedeutung. Sie kompensiert natürliche Ressourcenarmut durch wissenschaftlich-technischen Reichtum. « ${ }^{4}$

Die Provokation der Realität liegt in der gut begründbaren Hypothese, daß das westdeutsche Wissenschaftssystem bis auf marginale Ausnahmen das ostdeutsche hätte auflösen und sich in den neuen Ländern hätte ausbreiten können; noch deutlicher: die westdentsche Wissenschaft hätte die ostdentsche nicht gebraucht. Die erfolgreiche westdeutsche Wissenschaftsorganisation hätte ihr Territorium erweitert, ansonsten wenig Neues.

Sozzal hätte genau dieses Verfahren einen wichtigen und relativ großen, aber nicht den wichtigsten und größten Bereich des Öffentlichen Dienstes betroffen. Die damit verbundenen Ungerechtigkeiten wären den in anderen Bereichen "abgewickelten " Betrieben vergleichbar gewesen. Die Provokation der Realität liegt in der Hypothese, daß von der intellektuellen Produktion her erhebliche Unruhe und Widerstand zu erwarten gewesen wäre und daß unkalkulierbare, jedenfalls ambivalente, Solidarisierungen aus dem Westen politische Behinderungen bei der Anpassung nach sich ziehen würden.

Kulturpolitzsch war zu bedenken, daß - wie überall - die Hochschulen und auch die Akademien, potentiell Stätten von Opportunismus ebenso wie von Dissidenz waren, und daß während der Übergangszeit nach 1989 nirgendwo sonst so intensive Austauschbeziehungen wie im Wissenschaftsbereich eingetreten waren, ausgenommen vielleicht Schriftsteller, Künstler und Geheimdienste.

\footnotetext{
2 Ulnch K. Preuß, Der Liquidationsvertrag, TAZ 14.09. 1991, S. 12.

3 Einigungsvertrag Art. 38 Abs. I S. I-2.

4 Wissenschaftsrat, Empfehlungen des Wissenschaftsrates zu den Perspektiven der Hochschulen in den goer Jahren, Köln 1990, S. 21
} 
Rechtlichs schließlich hat die Wissenschaftsverfassung der Bundesrepublik selbst die Grenzen der Unterwerfung bzw. Entfaltung gesetzt. Der diktatorisch gemeinte Satz des Bundes Freiheit der Wissenschaft: „Ehe die Hochschulen autonom werden können, müssen sie autonomiefähig sein. Die neuen Länder werden in einigen Jahren daran gemessen werden, w1e erfolgreich dieser Prozeß gelaufen ist «, ${ }^{6}$ läßt sich auch kontrafaktisch lesen: Daß die friedliche Umwälzung der DDR in der Wissenschaft ihre Fortsetzung finden könnte, wenn sie dort schon kaum ihren Ausgang genommen hatte. Nun ist aber die Inkorporation der DDR aus einem politischen in einen verwaltungsrechtlichen Prozeß übergeleitet worden, bevor die Politik der friedlichen Revolution im Osten die "Kollusion « (Maaz) mit dem Westen in einem interkulturellen Prozeß aufheben konnte. Es hätte nahegelegen, die Verfahren der "Selbstreinigung " (Ost) und der "Integrationsbereitschaft « (West) sich entwickeln zu lassen. Dann wäre nicht geschehen, was mittlerweile Realität ist: die juristische Collage aus Vergangenheitsbewältigung, Adoption der Organisation und fachlicher Dominanz durch den Westen.

\section{Rabmenbedingungen}

Dic Neologismen: Abwicklung, Warteschleife, Abberufung, Ehrenkommission... sind typisch für eine Semantik, die als Quid pro quo einen einfachen Sachverhalt verschleiern soll. Dieser besteht in der Schwierigkeit, das Inkorporierte in den Stoffwechsel des neuen Staates einzubringen. Das westliche Wissenschaftssystem ist auch nicht mehr, was es vor 1990 war. Es ist - wie andere Bereiche auch -

S Die wıchtıgsten Rechtsgrundlagen finden sıch für alle neuen Länder in:

- Einıgungsvertrag vom 31.8.1990; bes. Art. 1 3, 37, $3^{8}$ und Anlage II, XV Abschnitt I1, XVI Abschnitt III (BGBI. II, S. 889);

- Verordnung über Hochschulen der DDR vom 18.9.1990-geplante Geltung bıs 30.6.1991 (GBI. I S. 1585 );

- Tarifverträge Ost (GMBI. Nr. 24 vom 16.8.1991);

Berlin:

- Beschlüsse des Senats Nr. 274/90 vom 19.12. 1990 und Nr. 275/90 von 22.12. 1990 (Überführung, Abwicklung);

- Ergänzung des Berliner Hochschulgesetzes (ErgGBerlHG) vom 18.7.1991 (GVBl, S. 176);

- Beschluß des VG Berlin vom 20. 2. 1991-7 A 25.91-, teilwerse aufgehoben durch Beschluß des OVG Berlin vom 6.6.1991 - $8 \mathrm{~S}_{76.91}$;

Brandenburg:

- Beschluß der Landesregıerung vom 17. 12.1990 (Überführung, Teilabwicklung);

- Brandenburgisches Hochschulgesetz vom 24.6.1991 (GVBI. S. I 56);

Mecklenburg-Vorpommern:

- Beschluß der Landesregierung vom 19. 12. 1990 (Übernahme, Abwicklung, Neugründung, Umstrukturierung);

- Hochschulerneuerungsgesetz (HEG) vom 19. 2. 1991 (GVBI. S. 34);

- Überleıtungsverordnung vom 24.4.1991 (GVBI. S. 138);

- Musterverfahrensordnung vom 24.6.1991;

Sachsen:

- Beschluß der Landesregierung vom 11.12.1990 (Neustrukturierung);

- Beschluß der Landesregierung vom 9. 1.1991 (Abwicklung)

- Richtlinıen des Sächsıschen StMWK zur Prüfung der persönlichen Integrı̈ät von Angehörıgen der Unıversı̈täten und Hochschulen (Einzelfallentscheıdungen) vom 7.5. 1991;

- Sächsısches Hochschulerneuerungsgesetz vom 25.7.1991 (GVBI. S. 261);

Sachsen-Anhalt:

- Beschluß der Landesregierung vom 18. 12. 1990 (Abwıcklung, Evaluatıon, Neustrukturıerung);

- Hochschulerneuerungsgesetz (HEG LSA) vom 31.7.1991 (GVBI. S. 198);

Thürnngen:

- Beschluß der Landesregierung vom 17.12.1990 (Neustrukturierung: Positivliste);

- Nebentätigkettserlaß des th. MWF vom 21.2.1991;

- Drittmittelforschungserlaß des th. MWF vom 21.2.1991;

- Vorläufiges Thürıngisches Hochschulgesetz vom 14. 5. 1991 (GVBI. S. 79);

- Evaluationsordnung für Thürıngische Hochschulen vom 6.7.199 I (GVB1. S. 130);

6 Bund Freiheit der Wissenschaft, Hochschulerneuerung in den neuen Bundesländern, in: Freiheit der Wissenschaft 3/1991, 3-4 
ökonomisch unter Druck und muß legitimieren, warum die besseren Standards

West die Unterwerfung der minderen Standards Ost rechtfertigen. Nun sind aber beide Wissenschaftssysteme, mit graduellen Differenzen, unvversalistzschen Wissenschaftskonzeptzonen unterworfen. Das bedeutet, daß die Niveauunterschiede unter dem kritischen Auge der internationalen Scientific Community und nicht mehr nur der nationalen Interessen ausgeleuchtet und bearbeitet werden. Das Zauberwort für Legitimation, vor allem der Auswechslung des Hochschulpersonals, heißt demnach Evaluation. Die Begutachtung durch den Wissenschaftsrat soll zur »Anpassung in die gemeinsame (!) Forschungsstruktur der Bundesrepublik Deutschland « führen (Art. 38 Abs. 2 Einigungsvertrag) - eine sprachliche Fehlleistung ohne weiteren Interpretationsbedarf. Evaluation soll die Objektivation der Ost-Behauptungen liefern, daß a) nicht "alles schlecht" war, und daß b) das wissenschaftliche (im Gegensatz zum ideologischen) Substrat der DDR übernehmbar sei. Das erklärt die große anfängliche Bereitwilligkeit von Teilen der DDR-Hochschulen und Akademien, sich evaluieren zu lassen.

Zum $\imath$ deolog $2 s c h e n$ Substrat verhält sich der Anpassungsvorgang höchst differenziert. Gerade weil marxistisch-leninistische Wissenschaft bloß eine Chiffre - und keine durchgängige "Weltanschauung - war, konnte sich hinter ihr fast jede Spielart von Wissenschaft in allen Konfigurationen verbergen. Das ging von überzeugter Propaganda, die niemanden überzeugen konnte, bis zu einem Vorwortopportunismus, hinter dem sich, gedeckt durch ein paar Parteitagszitate, rüdester Positivismus versteckte. Die in den Alltag übernommenen Begriffe der politischen Selbstreinigung und Säuberung finden auf mehreren Ebenen ihre Entsprechung: individuell (Einzelfallprüfung), institutionell (Institute, ganze Hochschulen) und informell durch Vermeidung von politischer Verantwortlichkeit in den Verwaltungsverfahren.

Die im folgenden beschriebenen Vorgänge und ihre Bewertung lassen sich nur verstehen, wenn die Rahmenbedingungen kurz erläutert werden:

- Das Wissenschaftssystem der DDR war zwar im Westen bekannt, aber nicht hinreichend gekannt. Die Wissenschaftsforschung glaubte, keinen Schwerpunkt bilden zu müssen, um die Rolle der DDR-Wissenschaft einschätzen zu können. Nachträgliche Defizitanalysen und Emeuerungsstrategien machen deutlich, daß die objektiven Differenzen noch einmal durch das kollusive Blockdenken in Abgrenzung zu und Anpassung an andere Systeme gebrochen wurden. So hat die »linke" westliche Betrachtungsweise die erkannten Defizite im Osten häufig unter einer apologetischen kritischen Solidarität verniedlicht, wenn zum Beispiel die schlechten Startbedingungen der DDR für alle Dysfunktionalitäten verantwortlich gemacht wurden. Umgekehrt hat die DDR-Wissenschaft, wenn westliche Vorsprünge evident und nicht zu verheimlichen waren, immer Gründe gefunden, warum sie in ihrem System nicht notwendig oder erstrebenswert sein sollten.

- Die Rolle der Akademie der Wissenschaften, ihr Verhältnis zu den Universitäten, deren Forschungskapazität, die realen Aufstiegsmuster und ihre ideologische Darstellung sind weitere Bereiche von westlichem Nichtwissen. Die hochschulpädagogische und hochschulwissenschaftliche Komparatistik, die teilweise über westliche hochschuldidaktische Einrichtungen oder das Zentralinstitut für Hochschulforschung in Ost-Berlin möglich gewesen wäre, hat wenig Breitenwirkung gehabt, weil die Schwerpunkte im Westen systemimmanent und EG-orieniert waren (Probleme wie Studienzeit, internationaler Austausch, Mobilität, Sozialbedingungen von Studium, Forschung und Lehre sind keine Themen für das östliche System gewesen). Die Wissenschaft in der DDR war de facto Ausland.

- Die Retsekader »erzählten«, was sie berichten durften, und prägten mit ihrer 
gezielten Anekdotik zusätzlich das westliche Bild. Aber diese privilegierten Boten des Systems brachten natürlich auch Information aus der Scientific Community West in den Osten, die ihnen einen Vorsprung vor denen sicherten, die, aus welchen politischen Gründen auch immer, nicht in den Westen reisen durften. Das erklärt sehr viele Startungleichheiten nach der Öffnung der Grenze.

- Die Rolle der DDR-Wissenschaft gegenüber und mit den östlichen realsozıalisttschen Ländern wurde im Westen überwiegend strategisch diskutırt. Die Sowjetunion erschien, abgesehen von ihrer politischen Bedeutung, nach 1985 zunehmend auch als der interessantere Partner. Andererseits gab es zu Beginn des Inkorporationsprozesses eine vage, ebenso strategische Hoffnung, über die wichtige Position der DDR im sozialistischen Block Einfluß auf die Wissenschaftslandschaft, vor allem im Forschungs- und Entwicklungsbereich, weit über die ostdeutsche Grenze hinaus zu erlangen.

\section{Evaluation}

Schon vor dem Einigungsvertrag war den wissenschaftlichen Einrichtungen der DDR, einschließlich den Hochschulen, ein objektivierbarer Vergleichsmaßstab durch die westdeutsche Wissenschaft nahegelegt worden. Unter dem Stichwort "Evaluation « konnten mehrere Interessen gebündelt werden: Viele Einrichtungen der DDR erhofften sich davon einen möglichst großen Anteil von Personen und Instiutionen, die des Weiterbestands oder der unmittelbaren Integration in neue Strukturen für wert befunden würden. Die westlichen Wissenschaftspolitiker erhofften sich von einem formalisierten Evaluationsverfahren einerseits die Legitimation für Entscheidungen bei gleichzeitiger Abwehr jedes Willkürverdachts, zum anderen würde die Evaluation ihnen quantitative Argunente für eine Reduzierung des alten östlichen Systems in die Hand geben; für einen Teil der Betroffenen konnte ein Evaluationsverfahren entweder fachliche Rehabilitation oder persönliche Integrität bescheinigen, selbstverständlich war das Risiko, beides nicht zugeschrieben zu erhalten, bekannt. Es gab vielfältige und in ihrer Konstitution und Abfolge noch nicht vollständig rekonstruierte Versuche bei einzelnen Hochschulen, Sektionen, Fächern und Einzelpersonen, während der Übergangszeit der Regierungen Modrow und de Maiziére durch Kontakte mit dem Westen und interne Vereinbarungen Evaluationsprozesse selbst in Gang zu setzen. Wie in anderen Bereichen der Einpassung mischen sich hier aufrichtige Erneuerungsansätze, selbstkritische Moratorien und blanker Opportunismus. Es ist müßig, darüber zu spekulieren, welche Konsequenzen ein hinreichend langer Zeitraum der Selbstevaluierung und Neugestaltung gehabt hätte, weil die induzierte Evaluationsmechanik ab Mitte 1990 keinen Spielraum mehr für wirkungsvolle Selbsterneuerung von unten bot. Im Juli 1990 haben die Regierungen von Bund und Ländern den Wissenschaftsrat bereits aufgefordert, die außeruniversitären Forschungseinrichtungen der ehemaligen DDR zu begutachten. Diese Funktion wurde im Einigungsvertrag bestärigt.

Mit dem Wissenschaftsrat sollte der Evaluationsprozeß wenigstens für die außeruniversitären Forschungseinrichtungen eine Evaluationsinstanz von hoher Dignität und Akzepranz erhalten. Die Hochschulen unterliegen anderen Überprüfungsregeln, sind aber vom Wissenschaftsrat indirekt stets beeinflußt. Der Wissenschaftsrat ist ein für die westdeutsche Wissenschafts- und Hochschullandschaft kontinuierlich tätiges Organ für Begutachtungen von Forschung, Lehre, Investitionen und Bautätigkeit, darüber hinaus haben seine Empfehlungen normativen und legitimatori- 
schen Charakter. Landesregierungen, die sich ohne guten Grund den Empfehlungen des Wissenschaftsrats demonstrativ verweigern, erleiden bei ihren Wissenschaftsprogrammen wenigstens psychologische Statuseinbußen. Diese relativ positive Einschätzung des Wissenschaftsrats gründet unter anderem darauf, daß er mit seinen grundsätzlichen Empfehlungen die wohl breiteste und differenzierteste Planungsfolie für die Hochschulpolitik fortschreibt. Diese Einschätzung darf aber nicht darüber hinwegtäuschen, daß der Wissenschaftsrat schon durch seine Konstruktion eine strukturkonservative und staatstragende Argumentationsweise bevorzugen muß und Reformen nur höchst verschlüsselt empfehlen kann. Die "Stellungnabmen $z u$ außeruniversitären Forschungseinnchtungen - allgemeiner Teil « vom Juli I99 I lesen sich wie eine nachgeholte Vertrauenserklärung für ein »faires Verfahren. Der Wissenschaftsrat argumentiert, wie er bei seinen Prüfungen in westdeutschen Institutionen seit Jahren argumentiert hat, und vergißt auch nicht die besonderen sozialen und strukturellen Bedingungen der DDR als berücksichtigenswert einfließen zu lassen. Auch mildert er die harsche Einpassungsforderung des Einigungsvertrages in allgemeiner Form $a b$, um brauchbaren Einrichtungen dort ihren Stellenwert zu belassen. Allerdings enthalten diese Stellungnahmen gerade das nicht, was für politische Entscheidungen am wichtigsten wäre: eine intelligible komparatistische Studie über die Situation der Forschung in beiden ehemaligen Ländern unter dem Aspekt ihrer staatlichen Zusammenfassung. Der bloße Hinweis, daß man auch Einrichtungen in den alten Ländern selbstkritisch überprüfen müsse, weil dort auch nicht alles in Ordnung sei, reicht hier nicht aus, vor allem wird kaum darauf eingegangen, ob und wieweit eine Veränderung der alten Forschungseinrichtungen durch ihre neue Rolle im neuen Staat induziert wird.

Mittlerweile liegt eine Reihe von detaillierten Stellungnahmen des Wissenschaftsrats vor, die einzelne außeruniversitäre Forschungsinstitute und Bereiche der Akademie der Wissenschaften betreffen. Diese Gutachten können zur Komparatistik durchaus herangezogen werden, ihre Empfehlungen sind überwiegend von einer sehr distanzierten Pragmatik. Exemplarisch für eine positive Überleitung in eine westliche Struktur kann die "Empfehlung zur Weaterführung des Instztuts für Hochenergzephysik" vom 25. I. 1991 als Teil des DESY angesehen werden. Die "Stellungnahme zu den Zentralinstituten für Molekularbiologie, Krebsforschung und Herz-Kreislaufforschung in Berlin-Buch"vom selben Tag mag jede wissenschaftliche Qualifikation für sich haben, aber sie liest sich doch wie eine im Westen für den Westen angefertigte Umstrukturierungsstudie. Auch die "Empfehlungen für Akademzevorhaben in den neuen Ländern und in Berlin " vom gleichen Datum stellen einen wichtigen Abschnitt der Evaluationstätigkeit dar, weil hier direkt die Überleitung des alten DDR-Akademiemodells in die westliche Struktur demonstriert wird. Hier werden längerfristige geisteswissenschaftliche Projekte daran gemessen, ob sie in die disziplinäre Fachkultur des Westens passen, und gegebenenfalls zur Weiterführung vorgeschlagen. Alle Empfehlungen enthalten neben einer Bewertung des Status quo Aussagen über Umfang, Ausstattung, Kooperationsbeziehungen und Profil der begutachteten Einrichtung.

Eine oberflächliche Betrachtungsweise könnte also von einer vorbildlichen, überwiegend von Experten der Scientific Community getragenen Integrationsleistung sprechen. In der Tat sind die fachlichen Aussagen des Wissenschaftsrats und seine Empfehlungen durchaus geeignet, die politische Planung und das wissenschaftliche Selbstverständnis in den untersuchten Einrichtungen zu stabilisieren und Ansätze von Binnendifferenzierung zu erzielen. Eine Reihe von Faktoren schwächt aber das positive Potential erheblich ab und legt neue Hypotheken für die Zukunft.

Die Hochschulen sind von dieser Art der Evaluation nicht betroffen, was der 
Wissenschaftsrat bedauert; sie werden in ihrer Entwicklung von ganz anderen, explizit politischen Überlegungen getragen. Wie problematisch die Evaluationsdichotome ist, hat der Wissenschaftsrat in seinen "Empfeblungen zur Forschung und Lebre auf dem Gebiet der Rechtswrssenschaften in den neuen Ländern " vom I3.3. I991 dargestellt. Die in den Hochschulen je nach Bundesland unterschiedlichen Formen von Evaluation und Neustrukturierung haben immer die offene Frage zu bewältigen, welche Teile ehemals außeruniversitärer Forschungseinrichtungen sinnvollerweise oder aus pragmatischen Überlegungen zu integrieren wären. In der Hochschulpolitik spielen zudem, anders als bei außeruniversitären Forschungsinstituten, noch andere Faktoren, wie Studenten, der wissenschaftliche Nachwuchs, der höhere Grad an Politisierungspotential usw. eine Rolle.

Schließlich ist die Frage, wie denn DDR-Wissenschaft »überhaupt « zu bewerten sei, ebenso zentral wie unbeantwortet. Aus der Behandlung dieser Frage leite ich die wesentliche Kritik an den derzeitigen Verfahren ab, wobei diese Kritik für Hochschulen und außeruniversitäre Institute gleichermaßen gitt. An der Dominanz westdeutscher Maßstäbe bei allen Formen der Beurteilung besteht kein Zweifel, unabhängig vom Ausmaß der Beteiligung ostdeutscher Wissenschaftler an den Verfahren. Prüfungsverfahren, auch wissenschaftliche Evaluation, stehen stets im Spannungsverhältnis der Ansprüche von »Gleichheit« und »Vergleichbarkeit«. Die erste Norm gebietet die Berücksichtigung der Umstände, die eine bestimmte Entwicklung und Qualität einer Institution bedingt haben und zeigt die Maßstäbe auf, unter denen ein Höchstmaß an »Gleichbehandlung " bei nicht vergleichbaren Tatbeständen möglich ist. Die Vergleichbarkeitsnorm dagegen sucht für alle beteiligten Subjekte ein Höchstmaß an vergleichbaren Handlungsbedingungen herzustellen, verzichten also auf eine Konturierung der wesentlichen Unterschiede.

Verfassung, Gesetze, Wissenschaftsdiskurs und politische Rhetorik der DDR machen die Fixierung der Unterschiede anscheinend einfach (aus der Sicht der DDR ließe sich das gleiche über die Bundesrepublik nicht mit dieser Eindeutigkeit sagen, weil die normativen Unschärfen gerade zur Effizienz westlicher Wissenschaftssysteme gehörten). Die einfachste Form, um Vergleichbarkeit festzustellen, wäre, die Übereinstimmung von Norm und Realität zu fingieren und diese Fiktion so weit wie möglich als Realität nachzuweisen. Dieses Verfahren hat man ja tatsächlich bei der Auflösung von Instituten für marxistisch-leninistische Philosophie oder Ökonomie angewandt. Der Generalverdacht trifft hier die Sozialwissenschaften, die Pädagogik, Jura und die Wirtschaftswissenschaften sowie alle unmittelbar politischen Dienstleistungsdisziplinen. Die andere Methode ist die scheinbar unpolitische des Rekurrierens auf universelle und weithin anerkannte Standards wissenschaftlicher Arbeit, vermittelt z. B. über Publikationen, Mitgliedschaften in internationalen Netzwerken, Effizienz von nachgewiesenen Entwicklungen, Patenten und Lizenzen. Der Objektivismus scheitert spätestens bei einer Untersuchung der Arbeitsbedingungen und der physischen und psychischen Repressionsmechanismen bzw. ihres individuellen Unterlaufens durch Einzelpersonen oder Gruppen. Gleichwohl wird er noch immer stark gegenüber den Naturwissenschaften und Ingenieursdisziplinen vertreten, um diesen zugleich das Signum relativ großer Politikferne verleihen zu können. Beide Zugangsweisen und auch Mischformen des Vergleichs müssen dann defizitär bleiben, wenn tatsächlich angestrebt ist, eine Integration der beiden Systeme zu einer einheitlichen Wissenschaftslandschaft zu befördern, bevor diese ihr gesamtstaatliches Interesse formuliert hat.

So, wie die Dinge laufen, scheint sich eine Eingangshypothese zu bestätigen, daß nämlich die westliche Wissenschaft die östliche nicht im eigentlichen Sinne »braucht«, sondern die Übernahmekosten unter Ausnutzung des größtmöglichen 
Wirkungsgrades so gering wie möglich halten möchte. Vor der westlich-demokratischen Legitimationsfolie, zugleich Legitimationsfalle, sind viele ostdeutsche Prozeduren in der Tat weder integrationsfähig noch verteidigenswert. Abgesehen von allen ideologischen Restriktionen und dem Qualitätsverlust durch unzugängliche Information sind hier zu nennen: die Karrieremuster, der ungenügende internationale Austausch, die Fiktion einer praxis- und umsetzungsorientierten Wissenschaft in einem definierten nationalen Interesse (einschließlich "Plan«) und alle demotivierenden Faktoren der gesellschaftlichen Stiuation, die in die Hochschulen hineinwirken.

Auf der anderen Seite ist sehr viel an guter Qualität und/oder an Widerständigkeit durch die relative Privilegierung der wissenschaftlichen Institutionen, die hohe Arbeitsplatzsicherheit und bestimmte Nischenfunktionen zu erklären. Diese gute Qualität hat aber teilweise ihren Markt bereits gefunden. Viele hochqualifizierte Mitarbeiter der Akademie der Wissenschaften haben diese schon frühzeitig nach der Grenzöffnung verlassen, um Arbeitsplätze im westlichen Ausland anzunehmen. Dieser Prozeß hat sich fortgesetzt, während über die Zukunft der Akademie der Wissenschaften verhandelt wurde. Auch ist nicht von der Hand zu weisen, daß die westdeutschen Spitzenforschungseinrichtungen der Max-Planck-Geselischaft und der Fraunhofer-Gesellschaften bei ihren Institutsübernahmen »Rosinenpicken « betrieben haben, wodurch die Integration und Übernahme des Erbes für die Koordinierungs- und Abwicklungsstelle für die Institute und Einrichtungen der ehemaligen Akademie der Wissenschaften noch schwieriger wird.?

Das durch die Reisekader und die Interlinearlektüre geprägte westliche Bild von der DDR-Wissenschaft kann in der Kürze der Zeit die Deformationen oder kreativen Potentiale nicht erkennen, die z.B. bei Wissenschaftlern auftreten, die an der Ausreise gehindert wurden oder durch dissidentes Verhalten bei gleichzeitiger wissenschaftlicher Brauchbarkeit stark unter Druck geraten sind. Die Aufarbeitung solcher Individualschicksale wiederum legt nahe, möglichst schnell von einer »Typik« oder einem repräsentativen Bewertungsstandard Abstand zu nehmen. Denn all solches ließe sich ja nur rechtfertigen, wenn der zugrundegelegte westliche normative Maßstab in den alten Ländern ein überaus großes Maß an realer Entsprechung hätte. Wir kennen aber alle die vielfältigen Mechanismen, mit denen die wissenschaftlichen Normen unterlaufen, verbogen oder verkehrt werden. Ich erinnere nur an die Rolle von Gutachten, disziplinären Seilschaften, Staatsintervention bei unliebsamen Listenreihungen und ähnliches. Unterhalb der Ebene großer politischer Intervention ist meine bisherige Erfahrung die, daß die Konkurrenzmechanismen und informellen Hierarchisierungen in der alten DDR genauso gelaufen sind wie in der alten BRD, allerdings einerseits unter wesentlich unerfreulicheren politischen Rahmenbedingungen, andererseits unter vergleichsweise geringerem ökonomischem Druck.

Die Interpretation der Evaluation kann also nur politisch sinnvoll sein, wenn man nicht von den sozialen, kulturellen und individuell-machtpolitischen Differenzierungen absieht und die Vergleichsmaßstäbe in das Wissenschaftskonzept einbezieht, von dem man meint, daß der neue deutsche Staat es verwirklichen sollte bzw. dabei ist, es zu verwirklichen. Fundamentale Änderungen aus Sicht der Bundespolitik oder vis à vis der EG sind nicht abzusehen, allerdings erhebliche materielle Verschiebungen in einzelnen Bereichen. Die Übernahme der DDR hat vor allem in den Hochschulen im Westen eine politische Position entscheidend geschwächt, die

7 Vgl. KAI - Koordinierungs- und Abwicklungsstelle für die Instıtute und Einrıchtungen der ehemaligen AdW der DDR, Informationen 27.8.1991; Bund demokratischer Wissenschaftlerinnen und Wissenschaftler: Evaluation der Evaluation. In: Forum Wissenschaft 4/90, I-XXIV. 
über die Frage von Lehrqualität, Überlastung und Nachrücksicherung 1988 und 1989 gerade erst wieder bundespolitische Bedeutung erhalten hatte.

\section{Hochschulen zwischen Abrencklung und Neustrukturierung}

Die Hochschulen und Universitäten der DDR standen von Anfang an im Licht einer sehr ambivalenten öffentlichen Diskussion. Auf der einen Seite galten sie überhaupt nach der 3. Hochschulreform von $1968 / 69$ als deformierende Kaderschmieden, auf der anderen Seite wurde ihnen gute berufsqualifizierende Arbeit bei kurzen Studienzeiten und erfolgreicher Didaktik attestiert. Vor der Vereinigung waren es vor allem konservative westliche Politiker wie Wirtschaftsminister Hausmann ${ }^{8}$ die auf eine erhebliche ökonomische Dynamisierung durch die gut ausgebildeten DDR-Absolventen setzten. Hoffnungen, daß große Lehrkapazitäten in der DDR Entlastung für den westlichen Teil Deutschlands schaffen könnten, konkurrierten mit der Auffassung, daß die schlechte Ausstattung eine rasche Adaption an westliche Kapazitätsberechnungen ohnedies nicht zulassen würde. Der Streit um die verschiedenen Formen der personellen Erneuerung und Selbsterneuerung blockierte vor und nach dem 3. Oktober 1990 viele Ansätze. Die Ausgangslage wurde dadurch kompliziert, daß unter den Regierungen Modrow und de Maiziére folgenschwere Maßnahmen zur Veränderung und Erweiterung des Status vieler Hochschulen getroffen wurden, so erhielten viele Universitätsrang und Promotionsrechte. Bei all dem war von vorneherein die Alternative klar, entweder eine HRG-Anpassung zum schnellstmöglichen Zeitpunkt mit größter Effizienz durchzuführen oder sich für zwei Hochschulsysteme in einem Land zu entscheiden, die sich langsam zueinander entwickeln würden und schließlich eine Integration zu neuen Bedingungen erfahren könnten. Die zweite Variante hatte jenen illusionären Hoffnungsgehalt, den allgemein die sozialen Versprechungen vor dem 2.Juli bzw. dem 3.Oktober 1990 erweckten. Mit der Hochschulverordnung kurz vor dem Einigungstag (1 8.9. 1990, Gültigkeit bis 30.6. I991 geplant) hatte sich die erste Variante weitgehend durchgesetzt. Damit wurde die Hoffnung, daß die einzelnen Länder auch im Sinne des Föderalismus eine dreijährige Übergangsphase zu einer teilweise autonomen und selbstbestimmten Entwicklung nutzen würden, zunichte gemacht. Konsequenterweise haben sich die Länder an die Konstruktion eigener Landeshochschulgesetze gemacht, die nurmehr in unwesentlichen Varianten vom HRG abweichen. Auf die besondere Situation von Berlin werde ich noch hinweisen.

\section{Hochschulüberführung}

Zur Zeit sind 8 Universitäten und 28 andere Hochschulen aus den neuen Bundesländern Mitglieder der Hochschulrektorenkonferenz, der Nachfolgerin der Westdeutschen Rektorenkonferenz. Eine Reihe von Hochschulen, die zum 3. 10. 1990 bestanden haben, sind bereits aufgelöst, darunter einige Pädagogische Hochschulen. Die Aufnahmeprozeduren, die sich über mehrere Sitzungen der HRK hinzogen, haben gezeigt, wie schwer sich das westdeutsche System tut, wenn die Maßstäbe, wie gezeigt, zwischen politischer, fachlicher und ökonomischer Polarität schwanken. So wurde beispielsweise die Bergakademie Freiberg erst im zweiten Anlauf unter die Universitäten (mit vollem Stimmrecht) aufgenommen. ${ }^{9}$

Entscheidend für den Status der Hochschulen ist, wieweit sie den Standards der

8 Minister Hausmann bes seiner Rede am 23-4.1990 in Oldenburg.

9 Hochschulrektorenkonferenz, Anlagen zur Ordnung der HRK V. 2. 
verschiedenen Hochschularten im Westen entsprechen, z. B. in Bezug auf Promotions- und Habilitationsrechte (teilweise erst in der Übergangszeit verliehen), Fachbreite usw. Auch gab es in der DDR keine Entsprechung zu den Fachhochschulen. Weitere Indikatoren für die Akzeptanz sind die Aufnahme in den DAAD, die DFG und andere bundesweit agierende Wissenschaftsorganisationen.

Die Erneuerung steht unter vier mit- und gegeneinander operierenden Tendenzen:

- Die politısche Reviston ("Entstalinisierung ist ein unvollkommener Ausdruck dafür) soll die Überführung der Hochschulangehörigen in die normative Wertordnung des westlichen Wissenschaftssystems erzielen und zugleich den ideologischen Überbau (z. B. Erziehungsziel der sozialistischen Persönlichkeit) revidieren.

- Die strukturelle Einpassung soll die Kompatibilität mit dem westlichen, föderalen System auch in Bezug auf Personalstruktur, Vergleichbarkeit von Hochschularten und Abschlüssen, Ausstattung und Standards gewährleisten.

- Die ökonomische Adaptıon soll die Hochschulfinanzierung und materielle Ausbaubasis sicherstellen; dazu zählt auch eine Reduktion echter und vermeintlicher Personalüberhänge vor allem in der Lehre.

- Die substantıelle Erneuerung schließlich, die diesen Namen verdient, soll die inhaltlichen Perspektiven der Hochschulen in den neuen Ländern unter Beteiligung der Betroffenen bewirken.

Alle vier Dimensionen haben umfangreiche Prozeduren der Verrechtlichung in Gang gesetzt. Ein zusätzliches Spannungsverhältnis ergibt sich daraus, daß die soziale Absicherung der ostdeutschen Wissenschaftler mit der Neueinstellung von westdeutschen (und gelegentlich ostdeutschen) Kollegen kollidiert, soweit diese Stellen substituieren sollen oder Fächer neu begründen und strukturieren.

Das Hochschulerneuerungsprogramm vom 24.5. 1991 versucht, diesen Prämissen vor allem unter dem Aspekt der strukturellen und der fachlichen Erneuerung gerecht zu werden, ist aber unterkritisch gering ausgestattet. 1,8 Mrd. DM werden anteilig vom Bund, den neuen Ländern und dem Programm »Aufschwung Ost« aufgebracht.

Nicht zu vernachlässigen ist die psychologische und soziale Komponente, daß neue Stellen im Osten den westlichen Arbeitsmarkt für Ersteinstellungen und statusverbessernden Aufstieg entlasten, wo die Nachwuchssituation nicht extrem angespannt ist. Daß dabei drittklassige Westwissenschaftler eine "Chance erhalten, ist eine in Kauf genommene Folge. Aufbaubeauftragte, oft taktlos »Gründungsdekane« o.ä. genannt, sollen Fachbereiche und Fächer strukturierend leiten, wenn diese total aufgelöst wurden oder in Gänze umstrukturiert werden sollen. Die einzelnen Länder gehen dabei - mit Ausnahme Berlins - relativ konform vor, wobei die Differenzen in der inhaltlichen Spezifikation der gesetzlichen und anderen rechtlichen Maßnahme liegen.

Die politzsche Überprüfung hat in einzelnen Hochschulen schon sehr früh zur Einsetzung von Ehrenausschüssen u. ä. in unterschiedlicher Zusammensetzung geführt. Wesentlich sind aber landeseinheitliche Regelungen, die genaue Kriterien für die Einzelfallüberprüfung und die Konsequenzen negativer Entscheidungen regeln. Das wohl ausführlichste Dokument dazu sind die Sächsischen »Rıchtlinıen zur Prüfung der persönlichen Integrität von Angehörigen der Universitäten und Hocbschulen (Einzelfallentscherdungen) v vom 7.5.1991. Sie geben einen detaillierten Katalog früherer Tätigkeiten und Verhaltensweisen, durch die u. a. auch andere Menschen zu Schaden gekommen sind. Auffällig ist, daß das nachfolgende Hochschulerneuerungsgesetz vom 25.7. I99 I die "Erneuerung fachliche und politische Elemente sehr viel weniger detailliert aufzählt $(\$ \mathbb{S} 75-8 \mathrm{I})$ und auf die obige Verord- 
nung nicht direkt Bezug nimmt. Das »Ehrenverfahren« gem. $\$ 2$ Hochschulerneuerungsgesetz von Mecklenburg-Vorpommern (19. 2. 1991) nimmt explizit nur auf Stasi-Aktivitäten Bezug. Eine spätere "Musterverfahrensordnung"vom 24.6. I99I weitet die Verfehlungskataloge aus $\left(\mathbb{S}_{3}\right)$. Die Differenzierungen sind oft nur in Nuancen zu erkennen und lassen ebensoviel Unsicherheit beim Staat wıe für die Betroffenen erkennen. Nirgendwo ist geregelt, in welcher Weise Betroffene ihre (frühen oder späten) Einsichten in politisch motivierte (und sozial oder familiär begründete) Verfehlungen aufarbeiten oder revidieren können. Das leistet einem problematischen Schematismus Vorschub. Schon vor dem 3. 10. 1990 sind über I 500 Hochschullehrer/-wissenschaftler aus politischen Gründen entlassen worden, wobei gerade in den ideologisch schwerbelasteten Institutionen eine hier besonders angebrachte Einzelfallprüfung offenbar weitgehend unterblieb. ${ }^{10}$

Meines Wissens nicht gesetzlich geregelt ist bisher die Rehabilitation der Opfer des Systems, die aus politischen Gründen ihre Stellen in der DDR verloren haben oder diskriminiert wurden.

Die strukturelle Einpassung wird durch die Ablösung der Hochschulverordnung durch endgültige oder vorläufige Landesgesetze in asymptotischer Bewegung zum Hochschulrahmengesetz vollzogen. Dabei sind folgende Verfahrensstränge von besonderer Bedeutung:

- Die Überfübrung der Personalstruktur; hier geht es u. a. um die "HRG-Konformität" von Mitgliedern in Beschlußgremien, vor allem solchen, die selbst Personalentscheidungen treffen oder vorbereiten. Das hängt eng mit den verschiedenen Überleitungsverfahren zusammen. Mecklenburg-Vorpommern hat z. B. eine Überlettungsverordnung (24.4.1991), in der nach $\$ 13$ der Kultusminister freihändig sogenannte "HRG-Professoren « berufen oder sie auf Antrag durch die zuständige Kommission ohne Beschlußfassung derselben ernennen kann. Auch in anderen Ländern ist die teilweise Überführung des Personals in Positionen, die mit der Personalstruktur des HRG übereinstimmen, eine besonders problematische Prozedur. Selbst wenn alle Tätigkeitsmerkmale für Professoren zutreffen, sind die Einstellungsvoraussetzungen immer Ergebnis einer administrativen und politischen Interpretation ex post. Daß dieses Verfahren generell zwei Klassen von Hochschullehrern schafft, ist klar, weil aus dem Westen berufene Professoren die HRG-Eigenschaften ex lege besitzen.

Die hohe Zahl von unbefristeten Stellen in dauerhaften Arbeitsverhältnissen erzwingt eine massive Umwertung von Stellen in befristete Steilen zur Förderung des wissenschaftlichen Nachwuchses und für wissenschaftliche Assistenten.

- Feststellung fachlicher Eignung mit personellen Konsequenzen; politische Unbedenklichkeit reicht für die strukturelle Erneuerung keineswegs aus. So hat schon die vorläufige Hochschulordnung vom 18.9. 1990 bei der Möglichkeit der Abberufung (hochschulrechtlicher Neologismus) neben politischen auch "weiche "Strukturgründe für die Entfernung aus dem Dienst genannt ( $\$ 51$ Abs. 2:« ...d) den Wegfall des Berufungsgebiets, e) bei fehlender Eignung nach grundsätzlicher inhaltlicher Änderung des Berufungsgebiets...).*

Ein Beispiel: Evaluiert wurden in Sachsen-Anhalt bisher nur die abgewickelten Teileinrichtungen der Hochschulen. Die Beschäftigten in anderen Bereichen werden erst ab dem 4. I. 1991 durch Personalkommesstonen gemäß $\$ \$ 64-68$ Hochschulerneuerungsgesetz des Landes Sachsen-Anhalt auf ihre Integrität überprüft. Die Mitglieder der Personalkommission sind bis Ende Oktober 1991 selbst noch nicht "gegauckt«. Das Ministerium hat nicht alle Vorschläge der Hochschulen für die

10 Vgl. BDWI, Eyaluation der Evaluation, 1990, S. XI. 
Personalkommissionen genehmigt, sondern auch eigene Vertreter eingesetzt (ermächtigt durch $₫ 66$ Hochschulerneuerungsgesetz). Diesen Kommissionen gehören unter anderem drei ständige Vertreter des öffentlichen Lebens an, was einen bescheidenen Ansatz zur "Veröffentlichung « der Verfahren darstellt.

Das gesamte Verfahren hat einen ambivalenten Charakter, weil Bedarfsargumente mit Eignungsargumenten vermischt werden. Das hat schon in westdeutschen Überleitungsbestimmungen große rechtliche Schwierigkeiten bereitet, weil - nach fachlich positiver Prüfung - das von der Haushaltszuweisung letztlich entschiedene Bedarfsargument die Bedarfsanmeldungen der Hochschulen überstimmt. Gleichzeitig ist hier ein Einfallstor für die fachliche Nivellierung wissenschaftlicher Besonderheiten der DDR-Systematik gegeben, weil Fächer dort einen anderen, nicht immer politisch motivierten und oft fachlich begründeten Zuschnitt hatten als im Westen. Bis hin zur Nomenklatur sind hier Auswirkungen für die Strukturplanung zu erwarten.

Für die Strukturplanung ist auch von Bedeutung, wie die quantitativen Verhältnisse als »Soll« geregelt werden, wenn die künftige finanzielle Ausstattung keinen großen Spielraum für das »Ist « läßt. Andererseits bietet die völlige Überalterung des Lehrkörpers aufgrund der überwiegend lange bestehenden Dauerarbeitsverhältnisse die Chance, durch Umwidmungen und Umschichtungen personalpolitische Entscheidungen zu treffen, von denen westliche Hochschulen nur träumen können. An dieser Stelle führe ich systematisch eine politisch hochinstrumentalisierbare Größe an: Die personelle Ausstattung der alten DDR-Hochschulen. Das im Westen drastisch verschlechterte Betreuungsverhältnis Lehrende/Studenten (von I975 I:12 auf heute $>_{1: 16)}$ hat den Kontrast zur DDR deutlich werden lassen, wo das Verhältnis rechnerisch 1:s betrug. Damit sind zwei einander äußerliche Faktoren affiziert: die Hochschuldidaktik und die Ausstattungskosten. Es ist keine Überraschung, wenn die hochschulpädagogische Bewertung der ostdeutschen Hochschulen bzw. der dortigen Lehrenden in vielfacher Hinsicht positiv ausfällt - z. B. im Bereich der Kommunikationskultur oder der persönlichen Ansprache. Viele Kritikpunkte, die die SPIEGEL-Umfrage 1989 - methodisch problematisch, aber in der Sache korrekt - aktualisiert hat, konnten für die DDR nicht zutreffen. Nun hätte nahegelegen, daß diese günstige Voraussetzung die ehemaligen DDR-Hochschulen gerade zum Experimentierfeld didaktischer Reformen für das gesamte Hochschulsystem prädestiniert; statt dessen hat selbst die Hochschulrektorenkonferenz den Abbau von Überkapazitäten undifferenziert gefordert. (Im ïbrigen gilt dieses Argument - mutatis mutandis - auch für andere Bereiche, wie etwa eine Reform der Lehrerausbildung)."

- Die finanzıelle Umstrukturterung setzt aber auch hier an: die Finanzlage der neuen Länder ist bekanntlich katastrophal und wird im Wissenschaftsbereich noch dadurch erschwert, daß diesen Ländern viele neue außeruniversitäre Forschungseinrichtungen "zuwachsen «. Hier schließt sich die Argumentation, die zu Beginn dieser Ausführungen ja auch auf den geringen gesamtstaatlichen Stellenwert der Hochschulausbildung hingewiesen hat.

Die Berliner Situation ist gegenüber den bisher beschriebenen Sachverhalten noch einmal komplizierter. Hier werden zwei inkompatible Systeme innerhalb eines nur teilweise »neuen « Landes zusammengeführt. West-Berlin war ein Wissenschaftsstandort von hoher Bedeutung, Ost-Berlin einer von außergewöhnlichen Privilegien und politischer Brisanz in der alten DDR. Allein die Humboldt-Universität, traditionsreiches Flaggschiff Unter den Linden, macht die Problematik deutlich:

I I Christa Händle und Wolfgang Nitsch, Integrierte Lehrerausbildung bieibt aktuell, Oldenburg 199 I. 
Wieweit kann sie neben der FU und der TU die dritte - oder gar »erste « Universität der neuen Hauptstadt werden? Das politische Interesse richtet sich besonders auf diese Universität, nicht zuletzt, weil ihr Rektor Heinrich Fink eine kämpferische Ausnahmeerscheinung in der Zunft ist und beharrlich auf dem Selbsterneuerungsrecht und der Fähigkeit seiner Universität dazu beharrt.

Ich will den Erneuerungsaspekt (als vierten der oben aufgeführten Systematik) am Beispiel der Humboldt-Universität Berlin darstellen. Die Universität hat sehr früh mit Ehrenkommissionen und Planungs-Kommissionen begonnen, sich selbst zu prüfen und zu regenerieren. Dabei wurde sie aber schon zu Zeiten des SPD/ GRÜNEN-Senats massiv behindert, weil sie immer in die Schere von mangelnder Glaubwürdigkeit (politisch) und unzureichender Organisationsreform (pragmatisch) geriet. Daß mehrere Fachbereiche (darunter Jura, Geschichte, Philosophie, Wirtschaft, Pädagogik) »abgewickelt« werden sollten, ohne daß die dort möglichen Erneuerungsprozesse wenigstens kreditiert wurden, hat zu heftigen rechtlichen Auseinandersetzungen geführt, die zu gerichtlichen Teilerfolgen führten. ${ }^{12}$ Aber das Kernproblem war, wie die selbstauferlegten Prozeduren mit einem politischen Senat, mittlerweile CDU/SPD, zu verhandeln wären, der eine reine Staatsaufsicht zur Bedingung des Erhalts der Universität machen wollte. Hochschulpolitisch war die Frage, wieweit eine Universität der DDR sich zum Sprachrohr der westdeutschen Autonomie und ihrer praktischen Verfahren machen durfte und konnte. Das m. E. sehr tragfähige Berliner Hochschulgesetz vom 12.10. 1990 wurde durch ein Ergänzungsgesetz modifiziert (ErgGBerlHG vom 18.7. 1991), bei dem die Strukturen der Humboldt-Universität einem strengen Staatsregime unterworfen wurden. In dieser schwierigen Situation hat die Humboldt Universität Berlin ihre Konstruktion durchgehalten und bis zu einem gewissen Grad erfolgreich auch im Kuratorium durchgesetzt: Ihre Personalstrukturkommissionen und die Zentrale Personalstrukturkommission haben, unter Mitwirkung externer, d.h. westdeutscher Mitglieder die qualitative und quantitative Neustrukturierung soweit vorbereitet, daß die vorgesehenen Massenentlassungen unterbleiben werden, eine Überleitungs- und eine Vorruhestandsregelung die rasche Neubesetzung vieler Stellen und die Fortführung des Lehrbetriebs möglich machen, und daß eine für den Senat von Berlin akzeptable Größenordnung erreicht wird. Die Verhandlungen über die Umsetzung dieser Beschlüsse, die das Kuratorium am 4.9. I991 mit großer Mehrheit als Planungsgrundlage angenommen hatte, sind im Gange, und es hat den Anschein, daß diese Linie auch beim Wissenschaftssenator Erfolg haben wird.

\section{Fazıt}

Die Hochschulpolitik hat sich noch nicht hinreichend auf das geänderte Koordinatensystem der neuen gesamtdeutschen Wissenschaftspolitik eingestellt; die Chancen zu einem langsamen Übergang mit zeitweiler Dualität der Systeme wurden nicht genutzt. Damit ist eine Entzerrung von politischer Reorganisation und fachlichstruktureller Erneuerung fast unmöglich geworden. Die Kritik an den Verfahren die auch die vielen besonderen Einzelfälle hervorhebt - ist selbst in den Ambivalenzen befangen, die der Einigungsprozeß unreif übernommen hat und nicht aufheben kann. ${ }^{13}$

12 Vgl. VG Berlin, Beschluß vom 20.2.1991, OVG Berlin, Beschluß vom 6.6.1991.

13 Diese Kritik kann dann besonders deutlich die Brüche aufzeıgen, wenn sie aus eınem nıcht realsozıalistısch fixıerten marxıstıschen Lager kommt und die Doppelbödigkeıt der westdeutschen Praxıs an der normatıven Rhetorik der Übernahme mißt: exemplarısch Peter Marcuse, Abwıcklung in East Germany. Renewal, Destalinization or Suppression? Workıng Papers of the Institute on East Central Europe 1/1991: Columbia University 1991. 
Rechtlich wird mittelfristig eine Totalreviszon des $H R G$ notwendig, weil zu viele regelungsbedürftige Probleme überkritisch geworden sind. Schwierige Fragen, wie die der Besoldungsanpassung, des Beamtenstatus für Professoren, der Rentenregelung sind in der Diskussion und werden hier, wiewohl von aktueller Bedeutung, nicht abgehandelt, weil sie systematisch nur Spezialfälle der allgemeinen Folgen der Einigung für den Öffentlichen Dienst sind.

\section{Thilo Weichert \\ Überprüfung der öffentlichen Bediensteten in Ostdeutschland}

\section{Rabmenbedingungen: personelle »Altlasten " und öffentlicher Dienst}

Das öffentliche Dienstrecht der Bundesrepublik Deutschland ist seit dem Anschluß der ehemaligen DDR am 3.10.1990 um ein Problem reıcher. Eine ähnliche Frage bewegte viele Menschen Ende der 4oer/Anfang der joer Jahre, als in den öffentlichen Dienst der jungen Bundesrepublik alte nationalsozialistische Funktionsträger drängten.' Inzwischen kann das Problem der Nazi-Richter und -Beamten ${ }^{2}$ als durch Zeitablauf wertgehend gelöst angesehen werden. ${ }^{3}$ Eine ähnliche Problemstellung holt uns nun ein. Sie macht es notwendig, den Umgang mit Menschen zu regeln, welche für das SED-Regime, zum Beıspiel für das DDR-Ministerium für Staatssicherheit (MfS), aktiv waren. In Anlehnung an einen anderen Rechtsbereich kann hier von personellen "Altlasten « $^{4}$ geredet werden.

Ähnliche Probleme bestehen auch in anderen Beschäftigungsbereichen, so für die von der Treuhand verwalteten Betriebe,' für den Bereich der parlamentarischen Politik ${ }^{6}$ oder für Rechtsanwälte. ${ }^{7}$

Gründe gegen die Wetterbeschäfttgung von belasteten Trägern des Staatsapparates

Der Umgang mit diesen "Altlasten ${ }^{8}$ hat zunächst ökonomische, soziale und politısche Dimensionen, ist aber letztendlich eine ethische Frage: Ist es moralisch zu vertreten, daß Menschen, welche unter Ausnutzung ihrer staatlichen Machtposition fundamentale rechtsstaatliche Prinzipien und Menschenrechte verletzt haben, in öffentlich-rechtliche Dienstverhältnisse eines freiheitlich-demokratischen Rechtsstaates eintreten, durch die sie erneut mit Macht und Einfluß über andere Menschen ausgestattet werden?

Ist es für ein Mitglied der Bürgerbewegungen der DDR, das heute z. B. wegen seines Engagements für cine umfassende Aufarbeitung der DDR-Geschichte vor Gericht

I Majer, ZRP 1991, 17 I.

2 ]ngo Müller, Furchtbare Jurısten, 1987, 203 ff., Rasehorn, Der Rıchter zwıschen Traditıon und Lebenswelt, 1989 , 54 ff.; vgl. BTDrs. 10/6 $566=\mathrm{DRiZ}_{1987}$, 113 ; Wassermann, Die nichterliche Gewalt, 1985 , 64 f.; zu Beamten AK-GG-Ridder 2. Aufl. 1989 Art. 33 Abs. 1-3, Rdnr. 27 ff.

3 Der Spiegel 21/1991 v. 20.5. 1991, 52: Die Probleme bereinigen sich biologisch.

4 Den Begriff verwendet u.a. GdP-Vors.' Lutz und GLKA-Pressespr. in: Bürgerrechte \& Polizeı 38 (1) 1991), 30, 35.

5 Wib $9 / 91-1 / 17$ v. 23.5. 1991, 3; BTDrs. $12 / 489$

6 Der Spiegel 25/1991, $81 \mathrm{f}$.

7 Dazu TAZ v. 10.5.1991, 6 u. 17. 5. 1991, 6; thür LTDrs. 1/404

8 Zur Diskussion Weiß, ZBR 1991, $18 \mathrm{f}$. 